\title{
OCORRÊNCIAS ADVERSAS COM MEDICAÇÃO EM UNIDADE DE TERAPIA INTENSIVA: ANÁLISE DA ADMINISTRAÇÃO DE SOLUÇÕES HIDROELETROLITICAS E ANTIBIÓTICOS *
}

\author{
ADVERSE OCCURENCES WITH DRUGS IN INTENSIVE CARE UNIT: ANALYSIS \\ OF THE ADMINISTRATION OF ELECTROLYTE SOLUTIONS AND ANTIBIOTICS
}

\author{
Simone Manenti** \\ Adriano Bezerra Chaves*** \\ Regiane S. Leopoldino*** \\ Katia Grillo Padilha****
}

MANENTI, S. et al. Ocorrências adversas com medicação em Unidade de Terapia Intensiva: análise da administração de soluçōes hidroeletrolíticas e antibióticos. Rev.Esc.Enf.USP., v.32, n.4, p. 369-76, dez. 1998.

\section{RESUMO}

O presente estudo teve por objetivos: 1) verificar a incidência de ocorrências adversas (OAs) com medicação relacionadas ao tempo de infusão das soluções hidroeletrolíticas e ao número de doses de antibióticos prescritos e administrados aos pacientes; 2) caracterizar a natureza dessas ocorrências. A investigação foi realizada em duas UTIs de um hospital geral do Município de São Paulo. Fizeram parte do estudo os dados contidos nos prontuários de 51 pacientes que estiveram internados naquelas Unidades no mês de Agosto de 1996. Quanto à caracterização dos pacientes, 60\% tinham idade acima de 60 anos, 58,8\% eram mulheres, 49,1\% permaneceram na UTI entre 1 e 5 dias e, ao saírem dela, 41,2\% foram para a Unidade de Cuidados Semi-Intensivos. Referente à incidência de OAs relacionadas ao tempo de administração das soluções hidroeletrolíticas e ao número de doses de antibióticos, constatou-se o não cumprimento das prescrições médicas dos pacientes, em $76,3 \%$ e $38,6 \%$, respectivamente. A maior freqüência de irregularidades quanto aos soros (60,2\%) foi referente a administraçäo em tempo menor que o prescrito (adiantamentos) $e$, no caso dos antibióticos, a redução do número de doses (85\%). Considerando-se tais irregularidades como ocorrências indesejáveis na prática da enfermagem na UTI, lá que se investir na busca de medidas preventivas dessas ocorrências.

UNITERMOS: Medicação. UTI. Ocorrências adversas. Enfermagem.

\begin{abstract}
The aims of this study were: 1) to verify the incidence of adverse occurences (AOs) with medication related to the time of electrolyte solutions infusion and the frequency of doses of antibiotics prescribed and administerid to the patients; 2) to characterize the nature of those occurences. The study was developed in two ICUs of a general hospital of São Paulo City. The population was composed by 51 patients that were in the ICUs in August of 1996 . Sixty percent of the patients were older than 60 years, $58,8 \%$ were women, $49,1 \%$ remained in ICU from 1 to 4 days and $41,2 \%$ went to the Intermediate Care Units after ICU. Regarding the incidence of AOs related to the time of administration of the electrolyte solutions and the frequency of doses of antibiotics the non execution of the patient's meclical prescriptions was verified in $76,3 \%$ and $38,8 \%$ respectively. The largest frequency of irregularies with the electrolyte solutions $(60,2 \%)$ was the infusion faster than the prescribed time followed by the reduction of the number of doses of antibiotics administered. Taking these into consideration we have to invest in preventive measures to reduce those occurrences.
\end{abstract}

UNITERMS: Medication. ICU. Adverse occurences. Nursing.

\footnotetext{
* Projeto financiado pelo $\mathrm{CNPq}$

** Bolsista PIBIC/CNPq

*** Bolsista de iniciação científica do CNPq - Processo n ${ }^{\circ}$ 52:0.840/5 - "Ocorrências iatrogênicas de enfermagem na UTI"

*** Orientadora - Prof. Dr. do Depto. de Enfermagem Médico-Cirúrgica da Escola de Enfermagem da USP
} 


\section{INTRODUÇÃo}

Dentre as atividades desenvolvidas pela enfermagem na Unidade de Terapia Intensiva (UTI), bem como em qualquer outra unidade hospitalar, a administração de medicamentos é uma das mais presentes e exige do profissional conhecimento científico e habilidade técnica que the possibilite atender o paciente com a máxima segurança.

No caso específico das UTIs, tendo em vista a gravidade do quadro clínico e instabilidade apresentada pelos pacientes, a quantidade de drogas prescritas é grande e diversificada, exigindo, portanto, o máximo de atenção para que não ocorram acidentes relacionados a esse procedimento. Sabese, no entanto, que apesar dos cuidados as ocorrências existem, o que torna o seu controle fundamental.

Para REVERT et al $^{13}$ as ocorrências adversas ( O.As.) com medicação tem sido descritas desde o início da época contemporânea e não foi extinta até os dias atuais, mesmo com o grande avanço técnicocientífico que o mundo tem vivenciado no decorrer de todo esse periodo. Afirmam esses autores que, nos últimos anos, tem-se observado nos meios de comunicação e nos serviços de atendimento à saúde importante aumento do número de reclamações, denúncias e protestos feitos pelos pacientes e famílias baseadas em supostos problemas envolvendo erros, imprudência e até negligência dos profissionais, no decorrer da prestação de assistência à saúde.

Nesse sentido, relacionado à questão da medicação, tem sido grande a preocupação dos profissionais no estudo dessas ocorrências e na proposição de protocolos de identificaçãa $0^{1,8,13,14,17,18}$ com vistas ao estabelecimento de medidas que previnam sua incidência, obviamente, dentro de um contexto maior de qualidade total nas instituições hospitalares.

Na Enfermagem, OGUISSO ${ }^{\prime \prime}$ em 1974 já ressaltava que a ocorrência de acidentes com medicação é o que deixa o profissional de enfermagem mais vulnerável, não só pelo tempo útil consumido nessa atividade, como também pela grande quantidade de drogas disponíveis e a diversificação quanto a dosagem e as vias de administração possíveis.

Corrobora essa constatação, estudo realizado por FORMINO et $\mathrm{al}^{5}$, junto a enfermeiros de UTIs onde se constatou que as O.As. envolvendo a administração de medicamentos foram as mais citadas como causa de iatrogenia.

Estudo mais recentemente realizado por PADILHA $^{12}$, sobre ocorrências iatrogênicas em cinco
UTIs do Município de São Paulo, demonstrou que a segunda maior freqüência desses eventos $(33,4 \%)$ foi relacionada à medicação, incluindo atrasos e adiantamentos na infusão de soros, aplicação de dosagem aumentada da droga e troca de via de administração, entre outros, evidenciando a presença de tais ocorrências no cotidiano das atividades de enfermagem na UTI, tanto naquelas de clínica médica, quanto cirúrgica.

Considerando especificamente a natureza das ocorrências com medicação na enfermagem, autores diversos apontam um leque considerável de possibilidades, citando dentre elas, a administração de drogas a pacientes trocados, omissão e excesso de doses, cálculos de dosagens errôneos, vias de administração incorretas e aplicação de drogas incompatíveis, o que acaba por expor pacientes e profissionais a um maior risco de acidentes e suas implicações ${ }^{2,4,6,8,16,17}$.

Um fato que chama a atenção e que no decorrer da assistência de enfermagem se observa com freqüência é que, muitas vezes, pelo fato das ocorrências adversas com medicação não desencadearem, de imediato, conseqüências indesejáveis aos pacientes ${ }^{10}$ acabam por gerar uma cultura de desconsideração quando ocorrências consideradas "menores" acontecem. Assim podemos dizer quanto às infusões de soros para reposição hidroeletrolítica e administração de antibióticos, que nem sempre atendem rigorosamente a prescrição feita. Dessa forma, encontram-se atrasos e adiantamentos de soros, bem como doses de antibióticos além e aquém das prescritas que, embora não acarretando repercussões mais graves, constituem problemas que necessitam ser sanados, pois, a terapêutica prescrita, com certeza, pautouse numa necessidade identificada e que deve ser atendida

Frente a essas considerações e acreditando que a monitorização de ocorrências adversas, quaisquer que sejam, cria condições para a implementação de ações efetivas que contribuirão para a melhor qualidade do cuidado e que as ocorrências adversas constituem importante indicador da qualidade da assistência ${ }^{1,8,18}$, julgamos pertinente a realização desse trabalho, entendendo, evidentemente, que o fato de se estudar um desvio na assistência não implica em desconsiderar os benefícios trazidos a todos que dela necessitam.

\section{OBJETIVOS}

- Verificar a incidência de ocorrência adversas com medicação em UTI, quanto: 
-tempo de infusão das soluções hidroeletrolíticas prescritas e administradas aos pacientes;

-número de doses de antibióticos prescritos e administrados;

- Caracterizar a natureza das O.As. referente ao tempo de infusão das soluções hidroeletrolíticas e às doses de antibióticos administradas.

- Comparar a incidência de ocorrências adversas com medicação em UTIs de clínica médica e cirúrgica.

\section{MATERIAL E MÉTODOS}

O presente estudo, retrospectivo, de natureza descritiva foi desenvolvido em 2 UTIs de pacientes adultos de um hospital público, localizado no Município de São Paulo, sendo designada como A a UTI cirúrgica e B, a UTI de Clínica Médica.

\subsection{Material}

Foram obtidos os dados registrados nos prontuários de 51 pacientes internados naquelas unidades no mês de agosto do ano de 1996 e que sofreram uma ou mais ocorrências adversas relacionadas à administração de soluções hidroeletrolíticas e/ou antibióticos.

Como ocorrência adversa considerou-se qualquer irregularidade referente ao tempo de infusão das soluções hidroeletrolíticas e ao número de doses de antibióticos prescritos e administrados.

\subsection{Coleta de dados}

A coleta de dados foi realizada pelos próprios pesquisadores que utilizaram os prontuários dos pacientes como fonte de informações.

\subsubsection{Instrumento}

Os dados foram obtidos por meio de uma ficha de coleta de dados elaborada para este estudo, sendo modificada após a realização do teste piloto que apontou necessidades de ajustes.

Tal instrumento foi composto por duas partes: a primeira abrangeu os dados de identificação do paciente (nome, idade, registro e sexo ) e dados referentes a internação na U'TI (causa da admissão, dias de permanência e saída da unidade com o respectivo destino). A segunda parte referiu-se ao levantamento de informações voltadas diretamente ao atendimento dos objetivos propostos e foi assim construída: numa folha, elaborou-se uma tabela contendo espaços em branco, onde se especificava, na coluna, a solução hidroeletrolítica prescrita, bem como o período de tempo em que deveria ser administrada (por exemplo, SG 5\% - $1000 \mathrm{ml}$ e $\mathrm{NaCl} 20 \%$ - $10 \mathrm{ml}$, de 12 em $12 \mathrm{hs}$ )e, nas linhas, horário do início e término da infusão reais, anotados de acordo com os registros diários feitos pela enfermagem no impresso da prescrição médica. Numa $2^{\mathrm{a}}$ folha, igual tabela foi montada, com espaço, na coluna, para a especificação dos antibióticos prescritos (tipo e número de doses) e nas linhas, para a anotação, por dia, do número de doses checadas pela enfermagem. Com essa configuração o instrumento permitiu levantar os dados sobre o cumprimento ou não, tanto da prescrição das soluções quanto dos antibióticos.

\subsubsection{Operacionalização da coleta de dados}

Inicialmente, foi solicitada a autorização formal à instituição para que os dados fossem coletados.

Ap6s a aprovação do projeto de pesquisa pela Comissão de Ensino e Pesquisa do Hospital, solicitou-se, em seguida, ao Serviço de Arquivo Médico e Estatístico (SAME) a listagem dos pacientes internados nas duas UTIs, em agosto de 1996, mês escolhido aleatoriamente para o estudo. De um total de 65 pacientes internados foi possível o acesso a 51 prontuários, sendo 21 de pacientes da UTI A e 30 da UTI B.

Os instrumentos de coleta de dados foram preenchidos pelos pesquisadores nas dependências do SAME, nos meses de abril, maio e junho de 1997 , de acordo com a disponibilidade dos pesquisadores.

\subsection{Tratamento estatístico}

Os dados foram analisados segundo a freqüência absoluta e percentual. As comparações estatísticas entre os dois grupos, foram realizadas pelo teste de Qui-quadrado $\left(\mathrm{X}^{2}\right)$, ao nível de significância de $5 \%$ e prova de Fisher, quando indicada ${ }^{15}$.

\section{RESULTADOS E DISCUSSÕES}

Para melhor elucidar os dados obtidos neste trabalho, os mesmos serão apresentados obedecendo a ordem estabelecida na ficha de coleta de dados, ou seja, caracterização da população da internação, e especificação das ocorrências adversas com soluções hidroeletrolíticas e antibióticos. 


\subsection{Caracterização da população.}

Do total de 51 pacientes que compuseram a população deste estudo, $21(41,2 \%)$ estiveram internados na UTI A (Cirúrgica) e $30(58,8)$ na UTI
B (Médica, sendo, em sua maioria, do sexo feminino $(58,8)$. Referente a faixa etária, os dados encontramse apresnetados na tabela a seguir.

Tabela 1: Distribuição dos pacientes das UTIs a e B, segundo a idade. São Paulo, 1997.

\begin{tabular}{|c|c|c|c|c|c|c|}
\hline \multirow[b]{2}{*}{ Pacientes } & \multicolumn{2}{|c|}{$\mathrm{A}$} & \multicolumn{2}{|c|}{ B } & \multicolumn{2}{|c|}{ TOTAL } \\
\hline & \multirow{2}{*}{$\mathbf{n}$} & \multirow{2}{*}{$\%$} & \multirow{2}{*}{$\mathbf{n}$} & \multirow{2}{*}{$\%$} & \multirow{2}{*}{$\mathrm{n}$} & \multirow{2}{*}{$\%$} \\
\hline Faixa Etária (anos) & & & & & & \\
\hline $\begin{array}{r}<40 \mid-60 \\
60 \mid-80 \\
80 \mid-100\end{array}$ & $\begin{array}{c}10 \\
8 \\
3\end{array}$ & $\begin{array}{l}47,6 \\
38,1 \\
14,3\end{array}$ & $\begin{array}{c}10 \\
12 \\
8\end{array}$ & $\begin{array}{l}33,3 \\
40,0 \\
26,7\end{array}$ & $\begin{array}{l}20 \\
20 \\
11\end{array}$ & $\begin{array}{l}39,2 \\
39,2 \\
21,6\end{array}$ \\
\hline Total & 21 & 100,0 & 30 & 100,0 & 51 & 100,0 \\
\hline
\end{tabular}

Na Tabela 1, verifica-se pelo teste do $\mathrm{X}^{2}$ que os dois grupos de pacientes não diferem quanto à idade e que a maioria $(60,8 \%)$ tem idade igual ou superior a 60 anos. Tais resultados são compatíveis com os encontrados na literatura e até esperados, tendo em vista o segmento cada vez maior de idosos na população brasileira e os conseqüentes agravos à saúde aos quais são suscetíveis e, que culminam com a internação em centros especializados de atendimento, no caso, as UTIs.

Quanto a causa da internação nessas Unidades, apesar da diversidade encontrada, os pósoperatórios de cirurgia do sistema digestivo (66\%) foram os mais presentes na UTI A e as insuficiências respiratória $(36,6 \%)$ e coronariana $(30 \%)$ na UTI B.

Tabela 2: Distribuição dos pacientes das UTIs A e B segundo a permanência na UTI. São Paulo, 1997.

\begin{tabular}{|c|c|c|c|c|c|c|}
\hline UTI & \multicolumn{2}{|c|}{ A } & \multicolumn{2}{|c|}{$B$} & \multicolumn{2}{|c|}{ TOTAL } \\
\hline Pacientes & \multirow{2}{*}{$\mathrm{n}$} & \multirow{2}{*}{$\%$} & \multirow{2}{*}{$\mathbf{n}$} & \multirow{2}{*}{$\%$} & \multirow{2}{*}{$\mathbf{n}$} & \multirow{2}{*}{$\%$} \\
\hline Permanência & & & & & & \\
\hline $1 \geq \mid-5$ & 14 & 66,7 & 11 & 36,6 & 25 & 49,1 \\
\hline $5 \mid-10$ & 2 & 9,5 & 9 & 30,0 & 11 & 21,6 \\
\hline $10 \mid-15$ & 1 & 4,7 & 5 & 16,7 & 6 & 11,7 \\
\hline $15 \mid-\geq 20$ & 4 & 19,1 & 5 & 16,7 & 9 & 17,6 \\
\hline Total & 21 & 100,0 & 30 & 100,0 & 51 & 100,0 \\
\hline
\end{tabular}

$X^{2} 3$ GL.obs. 6,20

$X^{2}$ crit. 7,81

Conforme pode-se observar na Tabela 2 , pelo teste do $\mathrm{X}^{2}$ não há evidência estatística para afirmar que os grupos diferem quanto ao tempo de permanência na UTI.

Analisando-se esse tempo, pode-se observar que $49,1 \%$ do total de pacientes nela permaneceram entre 1 e 4 dias. Apesar disso, verifica-se, pela análise isolada das Unidades que, enquanto na UTI cirúrgica $66,7 \%$ dos pacientes ficaram internados até 4 dias, na UTI de clínica Médica, quase a mesma porcentagem, ou seja, $63,4 \%$ nela permaneceram por um período maior de tempo, o que pressupõe um maior agravo à saúde dos pacientes desta Unidade. 
Tabela 3: Distribuição dos pacientes das UTIs A e B, segundo o destino após a saída da Unidade. São Paulo, 1997.

\begin{tabular}{|c|c|c|c|c|c|c|}
\hline \multirow[b]{2}{*}{ Pacientes } & \multicolumn{2}{|c|}{ A } & \multicolumn{2}{|c|}{ B } & \multicolumn{2}{|c|}{ TOTAL } \\
\hline & \multirow{2}{*}{$\mathbf{n}$} & \multirow{2}{*}{$\%$} & \multirow{2}{*}{$\mathrm{n}$} & \multirow{2}{*}{$\%$} & \multirow{2}{*}{$\mathrm{n}$} & \multirow{2}{*}{$\%$} \\
\hline Destino & & & & & & \\
\hline $\begin{array}{c}\text { UCSI }^{*} \\
\text { UI }^{* *} \\
\text { outro hospital } \\
\text { obito }\end{array}$ & $\begin{array}{l}14 \\
2 \\
1 \\
4\end{array}$ & $\begin{array}{c}66,7 \\
9,5 \\
4,7 \\
19,1\end{array}$ & $\begin{array}{c}7 \\
7 \\
2 \\
14\end{array}$ & $\begin{array}{c}23,3 \\
23,3 \\
6,7 \\
46,7\end{array}$ & $\begin{array}{c}21 \\
9 \\
3 \\
18\end{array}$ & $\begin{array}{c}41,2 \\
17,7 \\
5,9 \\
35,2\end{array}$ \\
\hline Total & 21 & 100,0 & 30 & 100,0 & 51 & 100,0 \\
\hline
\end{tabular}

O teste do $\mathrm{X}^{2}$ aplicado aos dados da Tabela 3 permite afirmar que os dois grupos diferem quanto ao destino após a saída da UTI. Nesse sentido, foi interessante notar que, embora $41,2 \%$ do total dos pacientes tenha recebido alta para a Unidade de Cuidados Semi-Intensivos (UCSI), tal fato parece ter ocorrido em função da UTI A, onde $66,7 \%$ tiveram esse destino. Verifica-se na UTI B que cerca da metade dos pacientes (46,7\%)foi a 6́bito, o que nos leva a inferir, ao associarmos a idade avançada e a maior permanência nessa Unidade (Tabelas 1 e 2) que o nível de gravidade desses doentes era considerável, quando comparados com aqueles da UTI A.

\subsection{Ocorrências Adversas com Soluções Hidroeletroliticas}

Tabela 4: Distribuição das soluções administradas aos pacientes das UTIs A e B, segundo o cumprimento do tempo prescrito. São Paulo, 1997.

\begin{tabular}{|c|c|c|c|c|c|c|}
\hline UTI & \multicolumn{2}{|c|}{$A$} & \multicolumn{2}{|c|}{$\mathrm{B}$} & \multicolumn{2}{|c|}{ TOTAL } \\
\hline Soluções & \multirow{2}{*}{$\mathrm{n}$} & \multirow{2}{*}{$\%$} & \multirow{2}{*}{$\mathbf{n}$} & \multirow{2}{*}{$\%$} & \multirow{2}{*}{$\mathrm{n}$} & \multirow{2}{*}{$\%$} \\
\hline Cumprimento do tempo prescrito & & & & & & \\
\hline Sim & 30 & 29,1 & 42 & 13,8 & 72 & 23,7 \\
\hline Não & 73 & 70,9 & 158 & 79,0 & 231 & 76,3 \\
\hline Total & 103 & 100,0 & 200 & 100,0 & 303 & 100,0 \\
\hline
\end{tabular}

$X^{2} 1$ GL. obs. 2,05

Pelos dados da Tabela 4 pode-se afirmar, com a aplicação do teste do $X^{2}$, que não houve diferença estatística entre os grupos no que se refere ao cumprimento do tempo de administração das soluções hidroeletrolíticas prescritas.

Os resultados obtidos dão mostras de uma realidade preocupante, uma vez que $70,9 \%$ das
Do total de 358 soluções administradas aos pacientes das duas UTIs, 55 não foram consideradas no estudo por conterem irregularidades no seu registro, resultando, portanto, 303 soluções administradas. Destas, $66 \%$ foram recebidas pelos pacientes da UTI A e $34 \%$ pelos da UTI B, observando-se, portanto, no decorrer da internação, uma média de 4,6 soluções administradas por paciente na UTI cirúrgica (A) e 6,6 na UTI Médica (B).

No que se refere ao tipo de solução, volume, composição e tempo de infusão, observou-se que as maiores porcentagens recaíram sobre a prescrição de solução glicosada (SG) a $5 \%(166 \cdot 54,2 \%)$, volume de $1000 \mathrm{ml}(180-58,8 \%)$, acrescidos de cloreto de sódio a $20 \%$ e cloreto de potássio a $19,1 \%$ (180. $58,8 \%$ ), para serem infundidos em 12 horas. soluções administradas na UTI A e $79,0 \%$ na UTI B não foram infundidas no tempo recomendado. Considerando-se a finalidade terapêutica dessas soluções pode-se dizer que, dadas as irregularidades observadas, as necessidades hidroeletrolíticas dos pacientes até podem ter sido atendidas, porém de forma inadequada, conforme dados da tabela a seguir. 
Tabela 5: Distribuição das soluções hidroeletrolíticas administradas aos pacientes das UTIs A e B, segundo a natureza da irregularidade no tempo de administração. São Pulo, 1997.

\begin{tabular}{|c|c|c|c|c|c|c|}
\hline UTIs & \multicolumn{2}{|c|}{ A } & \multicolumn{2}{|c|}{$\mathrm{B}$} & \multicolumn{2}{|c|}{ TOTAL } \\
\hline Soluções & \multirow{2}{*}{$\mathrm{n}$} & \multirow{2}{*}{$\%$} & \multirow{2}{*}{$\mathrm{n}$} & \multirow{2}{*}{$\%$} & \multirow{2}{*}{$\mathbf{n}$} & \multirow{2}{*}{$\%$} \\
\hline Irregularidade na Administração & & & & & & \\
\hline Adiantamento & 46 & 63,1 & 93 & 58,9 & 139 & 60,2 \\
\hline Atraso & 27 & 36,9 & 65 & 41,1 & 92 & 39,8 . \\
\hline Total & 73 & 100,0 & 158 & 100,0 & 231 & 100,0 \\
\hline
\end{tabular}

Na Tabela 5, verifica-se pelo teste do $X^{2}$ que os dois grupos não diferem quanto a natureza das irregularidades ocorridas na administração das soluções hidroeletrolíticas.

Conforme demonstrado, $60,2 \%$ das irregularidades referem-se à administração das soluções em tempo menor do que o prescrito, configurando os "adiantamentos dos soros", denominação comum utilizada entre os profissionais de enfermagem. Por outro lado, os atrasos, ou seja, a infusão das soluções em tempo maior que o prescrito ocorreu em $39,8 \%$ das soluções administradas. Referente a essas soluções, GIROTTI et al ${ }^{7}$, em estudo realizado sobre erros na administração de medicamentos na UTI encontrou uma maior incidência daqueles relacionados a terapêutica eletrolítica e ácido-básica, cuja diferença estatística foi significante quando comparada com as demais drogas utilizadas.

Apesar de não ser objetivo deste estudo explorar as causas que levaram a tais ocorrências, há que se considerar, numa análise mais abrangente, que inúmeros fatores estão envolvidos nessas situações ${ }^{12}$. Dentre eles, pode-se incluir desde aqueles que envolvem infra-estrutura de recursos humanos e de equipamentos até os relacionados ao tipo de acesso venoso disponível, quantidade desses acessos e condições clínicas do paciente.

Do ponto de vista de recursos humanos, estudo realizado por MIYADAHIRA et al $^{9}$ demonstrou que a relação numérica profissional de enfermagem/ paciente, mais especificamente do auxiliar de enfermagem, nas UTIs do Município de São Paulo, foi da ordem de 1:2 e 1:3. Tal relação, até considerada satisfatoria para os padrões brasileiros, nem sempre o é, de fato, diante das demandas de cuidados dos pacientes. Sabe-se, além disso, que a escassez quantitativa de recursos humanos na enfermagem é um problema crônico, que se reflete também nas UTIs, apesar da complexidade dos doentes graves.

Quanto à disponibilidade de equipamentos nessas unidades, no caso, bombas de infusão, recursos indispensáveis para garantir a administração de vários soros e drogas de maneira segura, CRUZ et al $^{3}$ encontraram a existência de 1,6 bombas de infusão, por leito, nas UTIs do Município de São Paulo. Frente às necessidades terapêuticas dos doentes críticos, essa quantidade nem sempre possibilita que todas as soluções e drogas sejam infundidas por meio desses recursos, exigindo um controle manual por parte dos funcionários, muitas vezes em número insuficiente, conforme já mencionado, o que contribui para um controle menos rigoroso, portanto, de maior risco.

Os acessos venosos disponíveis no paciente para a administração da terapêutica hidroeletrolítica constituem também fatores dificultadores para a manutenção da velocidade correta. Muitas vezes, a presença de uma única via de acesso para administração concomitante de 4 ou mais soros e medicamentos, acaba por levar às irregularidades encontradas, tais como os atrasos evidenciados em quase $40 \%$ das soluções administradas. Nesse sentido, observou-se nos pacientes deste estudo que $92 \%$ tinham uma única via de acesso venoso para toda a terapêutica medicamentosa prescrita. Chamou a atenção o fato de apenas $46 \%$ dos pacientes terem acesso venoso central. Em igual porcentagem, os pacientes apresentavam um único acesso venoso periférico o que torna quase impossível manter a velocidade de infusão regular, sobretudo, quando há vários medicamentos associados. Verificou-se, por exemplo, o caso de um paciente da UTI $B$, com insuficiência respiratória que recebia por meio de uma única veia periférica cinco itens da prescrição médica.

Independente, porém, das causas que levaram a tais irregularidades, os resultados obtidos nos levam a pensar nas repercussões dessas ocorrências nos pacientes críticos, onde são freqüentes as insuficiências de múltiplos orgãos, destacando-se aqui as cardíacas, renais e respiratórias e a vulnerabilidade dos pacientes frente a volumes infundidos inadequadamente, sobretudo no caso dos "adiantamentos". Da mesma forma, deixa evidente que medidas preventivas podem ser adotadas, incluindo desde o treinamento de pessoal até o provimento de infra-estrutura adequada para $o$ funcionamento das UTIs. 


\subsection{Ocorrências Adversas com Antibióticos}

Quanto às ocorrências adversas relacionadas aos antibioticos, ou seja, com a quantidade de doses

prescritas e administradas, os resultados encontramse apresentados na tabela a seguir.

Tabela 6 - Distribuição das doses de antibióticos administrados aos pacientes das UTIs A e B, segundo a concordância com o número de doses prescritas. São Paulo, 1997.

\begin{tabular}{|c|c|c|c|c|c|c|}
\hline UTIs & \multicolumn{2}{|c|}{ A } & \multicolumn{2}{|c|}{$\mathrm{B}$} & \multicolumn{2}{|c|}{ TOTAL } \\
\hline Doses & \multirow{2}{*}{$\mathbf{n}$} & \multirow{2}{*}{$\%$} & \multirow{2}{*}{$\mathbf{n}$} & \multirow{2}{*}{$\%$} & \multirow{2}{*}{$\mathrm{n}$} & \multirow{2}{*}{$\%$} \\
\hline Concordância & & & & & & \\
\hline $\begin{array}{l}\text { Sim } \\
\text { Não }\end{array}$ & $\begin{array}{l}19 \\
11\end{array}$ & $\begin{array}{l}63,3 \\
36,4\end{array}$ & $\begin{array}{l}24 \\
16\end{array}$ & $\begin{array}{l}60,0 \\
40,0\end{array}$ & $\begin{array}{l}43 \\
27\end{array}$ & $\begin{array}{l}61,4 \\
38,6\end{array}$ \\
\hline Total & 30 & 100,0 & 40 & 100,0 & 70 & 100,0 \\
\hline
\end{tabular}

X 1 GL. obs. 0,00

$X^{2}$ crit. 3,84

Pela aplicação do teste do $\mathrm{X}^{2}$ aos dados apresentados na Tabela 6 , pode-se dizer que não há diferença estatística entre os grupos com relação à concordância entre o número de doses de antibióticos prescritos e administrados.

Constata-se pelos dados encontrados que, de um total de 70 doses administradas aos pacientes das duas UTIs, a maioria, isto é, $43(61,4 \%)$ foi realizada corretamente, conforme a determinação da prescrição médica. Apesar desse dado representar a maioria, esperava-se um cumprimento da prescrição de cem por cento face as particularidades dessas drogas. Observou-se, no entanto, que houve irregularidades em $27(38,6 \%)$ delas, cuja natureza encontra-se apresentada na tabela a seguir.

Tabela 7 - Distribuição das doses de antibióticos administradas aos pacientes das UTIs A e B, segundo a natureza da irregularidade. São Paulo, 1997.

\begin{tabular}{|c|c|c|c|c|c|c|}
\hline \multirow[t]{2}{*}{ UTIs } & \multicolumn{2}{|c|}{$\mathrm{A}$} & \multicolumn{2}{|c|}{$\mathrm{B}$} & \multicolumn{2}{|c|}{ TOTAL } \\
\hline & \multirow{2}{*}{$\mathbf{n}$} & \multirow{2}{*}{$\%$} & \multirow{2}{*}{$\mathbf{n}$} & \multirow{2}{*}{$\%$} & \multirow{2}{*}{$\mathrm{n}$} & \multirow{2}{*}{$\%$} \\
\hline Natureza da Irregularidade & & & & & & \\
\hline $\begin{array}{l}\text { Redução do } n^{\circ} \\
\text { Aumento do } n^{\circ}\end{array}$ & $\begin{array}{c}15 \\
1\end{array}$ & $\begin{array}{c}93,7 \\
6,3\end{array}$ & $\begin{array}{c}13 \\
3\end{array}$ & $\begin{array}{l}81,2 \\
18,8\end{array}$ & $\begin{array}{c}23 \\
4\end{array}$ & $\begin{array}{l}85,0 \\
15,0\end{array}$ \\
\hline Total & 16 & 100,0 & 16 & 100,0 & 27 & 100,0 \\
\hline
\end{tabular}

$p=0,29$

Após aplicação do teste de Fisher verifica-se que não há diferença estatística entre os grupos para afirmar que diferem quanto a natureza das irregularidades na administração dos antibióticos.

Analisando-se as 27 irregularidades encontradas, pode-se constatar que $23(85 \%)$ foram referentes a redução do número de doses, enquanto que $41(15 \%)$ implicaram num aumento desse número. Estudo realizado por GIROTTI et al ${ }^{7}$ sobre erros relacionados a administração de medicamentos na UTI, também apontou a omissão de doses de drogas e a administração fora do horário determinado como as principais causas de erro nessa categoria terapêutica. Um dado que cabe comentar quanto a redução do número de doses, é que seja possível que a administração correta tenha ocorrido, havendo apenas omissão na checagem do procedimento.

Outro aspecto observado foi que 12 doses deixaram de ser administradas em função da droga não estar disponível no hospital. Esse resultado vem corroborar afirmações feitas por vários autores ${ }^{1,6,12}$ que atribuem as ocorrências indesejáveis também a problemas de outros serviços, transcendendo a participação única do profissional envolvido no cuidado direto. Por essa razão, existe a recomendação do envolvimento da equipe multidisciplinar em estudos sobre ocorrências adversas com medicação, conforme 
citado por FUCQUA; STEVENS ${ }^{6}$; BECHTEL et al $^{1}$, LARRABEE et $\mathbf{a l}^{8}$.

Em sintese, os resultados desta investigação mostram a importância do levantamento das ocorrências indesejáveis na prática da enfermagem como caminho para a ruptura numa cadeia de eventos que comprometem a recuperação do doente crítico, porém, indicam também a necessidade de estudos que avancem na busca das causas que as originam e nas medidas de intervenção que as previnam.

\section{CONCLUSÕES}

Os resultados obtidos no presente estudo permitiram as seguintes conclusões:

- Referente a incidência de O.As. relacionadas ao tempo de infusão das soluções hidroeletrolíticas prescritas e recebidas pelos pacientes, verificou-se que $76,3 \%$ das soluções não foram administradas de acordo com o tempo estabelecido na prescrição médica.

- Quanto a incidência de O.As. referente ao número de doses de antibioticos prescritas e administradas, observou-se que não houve concordância em $38,6 \%$ do total de doses prescritas.

- Relacionada a natureza das O.As. referentes ao tempo de administração das soluções constatouse que a maioria, ou seja, $60,2 \%$ foi de adiantamentos do tempo de infusão; quanto aos antibióticos, $85 \%$ das irregularidades foram decorrentes da redução do número de doses administradas.

- A análise comparativa entre UTIs de clínica médica e cirúrgica evidenciou que não houve diferença estatisticamente significativa entre os dois grupos quanto a incidência e natureza das ocorrências adversas relacionadas ao tempo de infusão das soluções hidroeletrolíticas e ao número de doses de antibióticos prescritos e administrados.

\section{REFERÊNCIAS BIBLIOGRÁFICAS}

BECHTEL, G.A. et al. A continuous quality improvement approach to medication administration. J.Nurs. Care Qual., v. 7, n. 3, p. $28-34,1993$.

CARVALHO, J.F de. Modelo operacional de enfermagem com enfoque preventivo. Braślia, Associação Brasileira de Enfermagem, 1983./Mimeografado/.
CRUZ, D.A.L.M. et al. Disponibilidade de equipamentos em unidades de terapia intensiva do Munićpio de Sao Paulo. Rev.Bras.Terap.Intens., v. 9, n. 2., p. 82-6, 1997.

FERRAZ, E.R.et al. Iatrogenia: implicações para a assistência de enfermagem. Rev.Esc.Enf.USP, v. 16, n. 2, p. 165-9, 1982.

FORMINO, A.A. et al. Iatrogenia em enfermagem: percepção da enfermeira de UTI. São Paulo, Escola de Enfermagem da Universidade de São Paulo, 1987. 20p. (Mimeografado).

FUCQUA, R.A.; STEVENS, K.R. What we know about medication errors: a literature review. J. Nurs. Qual. Assur., v. 3, n. 1, p. $1-17,1988$.

GIROTTI, M.J. et al. Medication administration errors in an adult intensive care unit. Heart \& Lung., v. 16, n. 4, p. 449-53, 1987.

LARRABEE, J.H.et al. Interdisciplinary monitoring of medication errors in a nursing assurance program. J. Nurs. Qual. Assur., v. 5, n. 4, p. 69-78, 1991.

MIYADAHIRA, A.M.K. et al. Recursos humanos na Unidade de terapia intensiva. São Paulo, Escola de Enfermagem da Universidade de São Paulo, 1997. (Mimeografado).

OFFERHAUS, L. Drug - induced iatrogenic disease: information risks and prevention. Iatrogenics,, v. 1, n. 1, p. 3-6, 1991.

OGUISSO, T. Aspectos legais da anotação de enfermagem no prontuário do paciente. Rio de Janeiro, 1974.116p. Tese (Livre - Docencia) - Escola de Enfermagem Ana Nery, Universidade Federal do Rio de Janeiro.

PADILHA, K.G. Des-cuidar: as representações sociais dos enfermeiros em UTI sobre as ocorrências iatrogênicas de Enfermagem. São Paulo, 1994. 188p. Tese (Doutorado) - Escola de Enfermagem, Universidade de São Paulo.

REVERT, F.B. et al. Error professional en los servicios de urgencia. Rev. Rol Enf., v. 1, $\quad$ n. 163, p. 77-82, 1992.

ROS, M.I.D. et al. Errores en la administracion de medicamentos. Rev, Rol de Enf., v. 2, n. 158, p. 67-9, 1991.

SIEGEL, S. Estatistica não-paramétricas . Såo Paulo, McGraw - Hill, 1975.

SCHOLZ, D.A. Establishing and monitoring and endemic medication error rate. J. Nurs. Qual. Assur., v. 4, n .2, p. $71-85,1990$.

WALTERS, J.A. et al. Developing and implementing a tool measure severity of medication errors. J.Nurs. Care Qual., v. 6, n. 4, p.33-43, 1992

WILLIAMS, A.D. Development and application of clinical indicators for nursing. J.Nurs. Care Qual., v. 6, n. 1, p. 1-5, 1991. 